\title{
高温隔热用微纳陶瓷纤维研究进展
}

\author{
张晓山 ${ }^{1}$, 王 兵 $^{1}$, 吴 楠 $^{2}$, 韩 成 $^{1}$, 吴纯治 ${ }^{1}$, 王应德 ${ }^{1}$
}

(国防科技大学 空天科学学院 1. 新型陶瓷纤维及其复合材料重点实验室; 2. 材料科学与工程系, 长沙 410073)

摘 要: 陶瓷纤维具有密度低、强度高、耐高温、抗氧化和耐机械震动性能好等优点, 是空天飞行器、核能发电和 化工冶金等热防护领域所需的关键高温隔热材料。传统陶瓷纤维直径粗 $(\phi>5 \mu \mathrm{m})$ 、脆性大、热导率高, 在实际隔热 领域应用中受到了极大限制。减小纤维直径, 制备微纳陶瓷纤维, 不仅有利于提高纤维力学性能, 还有望改善其高 温隔热性能, 近年来引起了研究者的广泛关注。从微纳陶瓷纤维中影响热传输(气体热传导、固体热传导和辐射传 热)的本征因素出发, 有针对地进行组成和结构优化, 进而改善其高温隔热性能, 是当前微纳陶瓷隔热纤维研究的 重点方向。本文结合国内外研究现状, 在介绍微纳陶瓷纤维隔热机理的基础上, 按照纤维的组成和结构特点将目前 微纳陶瓷隔热纤维分为三类, 即微纳陶瓷纤维气凝胶、中空/多孔微纳陶瓷纤维和复合微纳陶瓷纤维。对这三类不 同特点的微纳陶瓷隔热纤维最新研究进展进行综述, 并展望了微纳陶瓷隔热纤维的未来发展方向。

关 键 词: 微纳陶瓷纤维; 高温隔热; 结构优化; 纤维气凝胶; 中空纤维; 复合纤维; 综述

中图分类号: TQ343 文献标识码: A

\section{Micro-nano Ceramic Fibers for High Temperature Thermal Insulation}

\author{
ZHANG Xiaoshan $^{1}$, WANG Bing ${ }^{1}$, WU Nan ${ }^{2}$, HAN Cheng ${ }^{1}$, WU Chunzhi ${ }^{1}$, WANG Yingde ${ }^{1}$
}

(1. Science and Technology on Ceramic Fibers and Composites Laboratory, College of Aerospace Science and Engineering, National University of Defense Technology, Changsha 410073, China; 2. Department of Material Science and Engineering, College of Aerospace Science and Engineering, National University of Defense Technology, Changsha 410073, China)

\begin{abstract}
Ceramic fiber has the advantages of low density, high strength, high temperature resistance and good mechanical vibration resistance. It is the critical high temperature thermal insulation materials especially in thermal protection fields such as aerospace vehicles, nuclear power plants and chemo-metallurgical industry, etc. The traditional ceramic fiber with large diameter $(>5 \mu \mathrm{m})$, high brittleness and high thermal conductivity has been greatly restricted in high temperature thermal insulation fields. In recent years, more and more attention has been paid to the preparation of micro-nano ceramic fibers by decreasing the diameter of fiber, which is not only beneficial to improve the mechanical properties of the fibers, but also to enhance their high temperature thermal insulation properties. Further, by finely regulating the composition and structure of the micro-nano ceramic fibers that intrinsically affecting the heat transfer (heat conduction of gas, heat conduction of solid and radiative heat transfer) mechanism in micro-nano ceramic fibers, the high temperature thermal insulation performance can be effectively improved, which is the current focus of the micro-nano ceramic fibers in high temperature thermal insulation fields. The thermal insulation
\end{abstract}

收稿日期: 2020-04-26; 收到修改稿日期：2020-06-09; 网络出版日期：2020-09-10

基金项目: 国防基础科研计划(XXXX2016550C001, XXXX2017550C001) National Defense Basic Research Program (XXXX2016550C001, XXXX2017550C001)

作者简介: 张晓山(1991-), 男, 博士研究生. E-mail: zhangxiaoshan15@nudt.edu.cn ZHANG Xiaoshan(1991-), male, PhD candidate. E-mail: zhangxiaoshan15@nudt.edu.cn

通信作者：王应德，教授. E-mail: wangyingde@nudt.edu.cn WANG Yingde, professor. E-mail: wangyingde@nudt.edu.cn 
mechanism of the micro-nano ceramic fibers was firstly introduced. Then, based on the research at home and abroad, this review divides the current micro-nano ceramic fibers into three categories according to the difference of their composition and structure, namely fibers aerogels, hollow/porous fibers and composite fibers. The latest research progress on composition and structure optimization of micro-nano ceramic fibers for high temperature thermal insulation is reviewed, and the future development tendency is prospected.

Key words: micro-nano ceramic fiber; high temperature thermal insulation; structural optimization; fiber aerogel;

hollow fiber; composite fiber; review

随着航空航天技术的不断发展, 高超声速飞行 器的飞行速度更高 ( $>5$ 马赫), 在大气层中飞行时间 更长, 飞行器迎风面和机翼前缘等部位气动加热严 重, 承受的热环境极为恶劣 ${ }^{[1-2]}$ 。当飞行器以 8 马赫 的速度在大气层中飞行时其头雉处的温度高达 $1793{ }^{\circ} \mathrm{C}$, 机翼前缘等部位温度达 $1455{ }^{\circ} \mathrm{C}^{[3]}$ 。为使 其内部仪器设备能在合适的温度范围内工作, 必须 采用高效的热防护系统。此外, 导弹和战斗机等武 器装备也对高性能耐高温隔热材料提出了迫切需求。

气凝胶材料具有较低的密度和热导率, 是目前 常用的隔热材料 ${ }^{[4-5]}$ 。但其力学性能低、脆性大, 且 在高温条件下其内部纳米孔结构易坍塌, 难以满足 航空航天等领域中瞬间热冲击和长时间承受高温等 极端环境应用要求 ${ }^{[6]}$ 。陶瓷纤维具有耐高温、抗氧 化、化学稳定性好和耐机械振动性能好等优点, 既 可作为气凝胶材料的增强体和红外遮光剂, 也可单 独作为高温隔热材料, 在航空航天、化工治金和核 能发电等领域具有广阔的应用前景 ${ }^{[7-8]}$ 。相比于传统 陶瓷隔热纤维 $(\phi \geqslant 5 \mu \mathrm{m})$, 细化纤维直径制备微纳陶
瓷隔热纤维 $(\phi<5 \mu \mathrm{m})$ 不仅有助于降低纤维热导率, 还能改善其力学性能, 近年来受到了广泛研究和关 注 $^{[9-11]}$ 。此外, 对微纳陶瓷纤维的组成和结构进行优 化, 可进一步改善其隔热性能, 是当前微纳陶瓷隔 热纤维研究的重点 ${ }^{[12-14]}$ 。本文结合国内外研究现状, 介绍了微纳陶瓷纤维及其隔热机理。在此基础上, 将微纳陶瓷隔热纤维根据其组成和结构特点分为了 微纳陶瓷纤维气凝胶、中空/多孔微纳陶瓷纤维和复 合微纳陶瓷纤维三类, 系统综述了这三类纤维的最 新研究进展(图 1), 并对其存在的问题进行分析总 结，展望了微纳陶瓷隔热纤维未来发展方向。

\section{1 微纳陶瓷纤维及其隔热机理}

高孔隙率的纤维隔热材料, 通常在大气环境中 应用, 纤维之间孔隙是由气体填充(图 2(a) II)。由于 纤维材料内部没有足够的气压差, 且纤维之间的孔 隙不贯通, 因而在微纳纤维隔热材料中对流换热很 小, 可忽略不计 ${ }^{[15-16]}$ 。在微纳陶瓷隔热纤维中, 热传

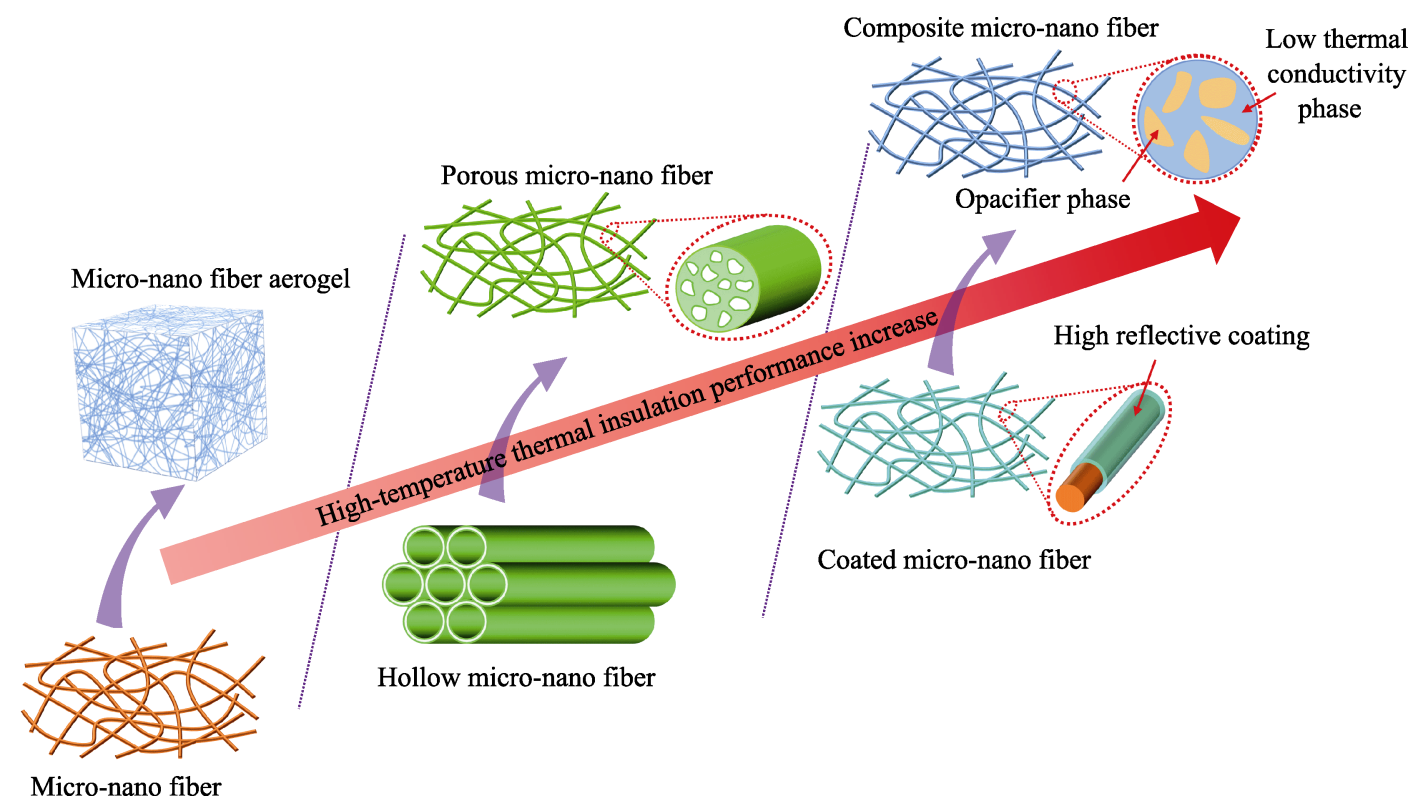

图 1 微纳陶瓷隔热纤维发展趋势示意图

Fig. 1 Schematic of development trend of the micro-nano ceramic fiber 

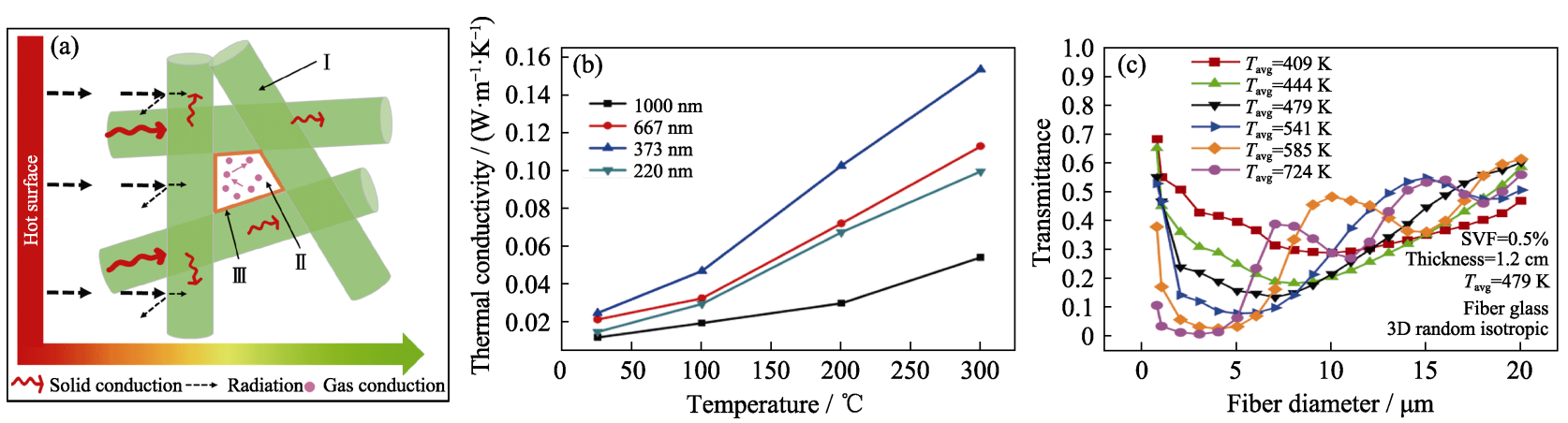

图 2 微纳陶瓷纤维热传导示意图(a), 不同温度下直径对碳纳米纤维热导率的影响(b)和 不同温度条件下不同直径纤维红外透过率(c) $)^{[13,21]}$

Fig. 2 Schematic of micro-nano fiber heat conduction (a), effect of fiber diameter on thermal conductivity of carbon nanofiber at different testing temperatures (b), and transmittance values for fibers with different fiber diameters at different operating temperatures $(c)^{[13,21]}$

输主要包括气体热传导、固体热传导和辐射传热。 微纳陶瓷隔热纤维中热传输示意图如图 2(a)所示。

纤维隔热材料中的气体热传导包括两部分，即 孔隙中的气体分子(图 2(a) II)相互碰撞进行热量传 递(气体自身热导率)和气体分子碰撞固体纤维表面 (图 2(a)III)进行的热量传递(气固热导率)。根据库森 理论, 有效气体热导率可由式(1)表示 ${ }^{[17-18]}$ :

$$
\lambda_{\mathrm{g}}=\frac{\lambda_{\mathrm{g}, 0}}{\theta+2 \psi \frac{\beta l_{\mathrm{g}, 0} f_{\mathrm{v}}}{P_{\mathrm{r}} \varepsilon d}}
$$

式中, $\lambda_{\mathrm{g}, 0}$ 为气体分子自身热导率, $\theta 、 \psi$ 和 $\beta$ 是与库森 数相关的参数, $P_{\mathrm{r}}$ 为普朗特数, $\varepsilon$ 为常数, $l_{\mathrm{g}, 0}$ 为气体 分子平均自由程, $f_{\mathrm{v}}$ 为纤维体积分数, $d$ 为纤维直径。

由式(1)可以看出, 通过增加气体分子平均自由 程、增加纤维体积分数或减小纤维直径可降低有效 气体热导率。其中, 增加气体分子平均自由程可通 过降低气体压力或使用平均自由程较大的气体替换 空气来实现, 但操作工艺复杂, 且成本较高, 在实 际应用中可行度不大。因而, 通过增加纤维体积分 数和减小纤维直径是降低气体热导率最简单有效的 方法。但纤维体积分数过高也会使纤维之间固体热 传导增加。因此在纤维隔热材料设计中应综合考虑, 选取合适的纤维体积分数。

对于无机陶瓷纤维，固体热传导(图 2(a) I )主 要是通过声子传热。固体中声子的运动性质决定着 固体热导率。根据运动理论, 陶瓷纤维的固体热导 率可由式(2)表示 ${ }^{[16]}$ :

$$
\lambda_{\mathrm{s}}=\frac{1}{3} C_{\mathrm{s}} v_{\mathrm{ph}} l_{\mathrm{s}}
$$

式中, $C_{\mathrm{s}}$ 为单位体积声子的定容比热容, $v_{\mathrm{ph}}$ 为声子 平均速度, $l_{\mathrm{s}}$ 为声子平均自由程。对于固体材料而言, 单位体积声子的定容比热容即是材料的比热容与密
度的乘积，声子平均速度近似等于声速。

由式(2)可以看出, 材料的固体热导率与声子的 平均自由程紧密相关。声子的平均自由程主要受以 下几个因素影响: 晶界和相界面对声子的散射、声 子与声子自身之间的散射, 以及材料中的缺陷和杂 质等对声子的散射 ${ }^{[19]}$ 。声子的散射作用越强，声子 的平均自由程越小。一般而言, 声子的平均自由程 越小, 材料的固体热导率也越低。因此, 对于陶瓷纤 维隔热材料, 可通过增加材料中缺陷浓度、杂质含 量和晶界数量等手段来减小声子平均自由程, 进而 降低固体热导率。

辐射传热是指温度高于绝对零度的物体通过红 外辐射线向外进行热量传递。辐射传热主要取决于 材料对红外辐射区电磁波的光学响应。纤维材料的 辐射热导率可由公式 3 表示 ${ }^{[16]}$ :

$$
\lambda_{\mathrm{r}}=\frac{16 k_{\mathrm{B}} n^{2} T^{3}}{3 \rho e}
$$

式中, $k_{\mathrm{B}}$ 为斯蒂芬玻尔兹曼常数, $n$ 为材料折射率, $T$ 为温度, $e$ 为消光系数, $\rho$ 为材料密度。消光系数是指 红外辐射通过材料后因散射和吸收作用而导致的辐 射能量的衰减程度。提高纤维的消光系数, 即红外 遮蔽性能, 可有效降低辐射热传导。

综上所述, 微纳陶瓷纤维的隔热性能与其组成 和结构密切相关。当纤维体密度相同时, 纤维越 细, 纤维间的孔隙尺寸越小, 对气体分子运动的限 制作用越大，则气体热传导越低。同时，纤维直径减 小, 则纤维间的接触点增多, 热量在纤维中传递时 经过的路径更为曲折, 遇到的界面屏障增多, 也使 得固体热传导减小。因而, 减小纤维直径有助于提 高纤维材料隔热性能。Gibson ${ }^{[20]}$ 和 $\mathrm{Wang}^{[21]}$ 分别研 究了玻璃纤维和碳纤维(图 2(b)) 直径对其热导率的 影响。结果均表明, 随纤维直径减小, 其热导率呈 
下降趋势。此外, Arambakam 等 ${ }^{[13]}$ 通过理论计算表 明, 减小纤维直径还有助于降低纤维的红外透过率 (图 2(c))。尤其是在高温条件下, 微纳纤维具有较低 的红外透过率, 辐射热导率较小。

此外, 细化纤维直径还可改善陶瓷纤维的柔 性。当纤维直径降低到纳米级时, 尺寸效应能够赋 予纤维较佳的柔性 ${ }^{[10-11,22]}$ 。传统陶瓷纤维直径较粗、 脆性大, 在实际使用过程中难以充分填充异形隔热 夹层, 其应用受到较大限制 ${ }^{[8,23-24]}$ 。纤维柔性的提高 可使其获得较好的填充贴合性能和折叠性能, 提高 其重复利用率, 在隔热领域具有显著优势。进一步, 纤维直径减小, 在纤维内部存在裂纹等缺陷的可能 性减小, 有利于提高其强度 ${ }^{[25-26]}$ 。因此, 减小纤维直 径制备微纳陶瓷纤维不仅可以改善其隔热性能, 还 有助于提高其柔性和强度, 是当前陶瓷隔热纤维发 展的重要方向。

在众多的陶瓷纤维中, 氧化物陶瓷纤维 $\left(\mathrm{ZrO}_{2}\right.$ 、 $\mathrm{SiO}_{2}$ 和 $\mathrm{Al}_{2} \mathrm{O}_{3}$ 等) 以其较好的抗氧化性能和较低的热 导率成为目前常用的隔热纤维。 $\mathrm{Si}$ 等 ${ }^{[27]}$ 制备了 $\mathrm{SiO}_{2}$ 纳米纤维, 并通过在先驱体中加入 $\mathrm{NaCl}$ 实现了纤 维之间连接, 有效提高了纤维膜的拉伸强度, 制备 的 $\mathrm{SiO}_{2}$ 纤维膜具有较低的热导率 $\left(0.0058 \mathrm{~W} \cdot \mathrm{m}^{-1} \cdot \mathrm{K}^{-1}\right)$ 。 $\mathrm{Mao}$ 等 ${ }^{[28]}$ 制备了不同直径的 $\mathrm{ZrO}_{2}$ 纳米纤维, 可将其 用作高温隔热材料。但 $\mathrm{SiO}_{2}$ 和 $\mathrm{ZrO}_{2}$ 纤维经 $1200{ }^{\circ} \mathrm{C}$ 热处理后, 纤维中晶粒长大明显, 使得纤维膜强度 和柔性显著下降，在更高温度应用受限 ${ }^{[27-28]} 。 \mathrm{Al}_{2} \mathrm{O}_{3}$ 纳米纤维以其较好的耐高温性能而备受关注 ${ }^{[29-30]}$ 。 Zhang 等 ${ }^{[29]}$ 制备了由 $\alpha-\mathrm{Al}_{2} \mathrm{O}_{3}$ 纳米片沿 [0001] 晶面堆 叠而成纳米纤维, 由于在纳米片之间形成了 $\mathrm{MgAl}_{2} \mathrm{O}_{4}$ 结晶抑制剂, 制备的 $\alpha-\mathrm{Al}_{2} \mathrm{O}_{3}$ 纳米纤维展现出较好 的耐高温性能 $\left(1400{ }^{\circ} \mathrm{C}\right)$, 在高温隔热领域具有较好 的应用前景。

近年来, 具有较好耐高温性能的钙钛矿陶瓷纤 维也是高温隔热纤维关注的重点。Yuan 等 ${ }^{[31]}$ 率先制 备了耐高温性能达 $1300{ }^{\circ} \mathrm{C}$ 的 $\mathrm{BaZrO}_{3}$ 纤维。该课题 组又制备了 $\mathrm{CaZrO}_{3}$ 纳米纤维, 该纳米纤维具有较 低的热导率 $\left(0.413 \sim 0.501 \mathrm{~W} \cdot \mathrm{m}^{-1} \cdot \mathrm{K}^{-1}\right)$ 和较好的耐碱 腐蚀和耐高温性能 ${ }^{[32]}$ 。

此外, 具有较高红外反射率的陶瓷纤维也可用 作高温隔热材料, 有效降低高温条件下辐射热传 导。研究人员利用 $\mathrm{Y}_{2} \mathrm{O}_{3}$ 自身高红外反射率的特点, 制备了 $\mathrm{Y}_{2} \mathrm{O}_{3}$ 纳米纤维, 该纳米纤维在近红外波段 内平均红外反射率高达 $92 \%$, 在高温条件下隔绝辐 射传热具有显著优势 ${ }^{[33]}$ 。

目前, 微纳陶瓷隔热纤维取得了较大发展, 制
备了一系列具有较好隔热性能的微纳陶瓷纤维。但 当前的微纳陶瓷隔热纤维强度相对较低, 且在高温 条件下晶粒长大导致强度下降、脆性增大, 长期工 作温度较低。因此, 如何提高微纳陶瓷隔热纤维的 强度和耐高温性能是其未来发展中需关注的重点。

\section{2 微纳陶瓷纤维气凝胶}

将微纳陶瓷纤维组装成轻质、高孔隙率的纤维 气凝胶有助于充分发挥纤维自身柔性和低热导率的 特性, 有望克服传统陶瓷隔热纤维脆性大、热导率 高等不足, 制备出具有较好力学性能的高效隔热材 料, 在高温隔热领域具有较大应用潜力。自东华大 学丁涁课题组 ${ }^{[34]}$ 成功制备出具有良好压缩弹性的 $\mathrm{SiO}_{2} / \mathrm{PAN}$ 复合纤维气凝胶以来, 纤维气凝胶引起了 学者们的广泛研究。近来, 该课题组以 $\mathrm{SiO}_{2}$ 纳米纤 维为基体, 采用嗍硅溶胶为粘结剂先驱体, 制备了 纤维之间由硽硅酸盐陶瓷连接的 $\mathrm{SiO}_{2}$ 纳米纤维气 凝胶(图 3(a))。得益于 $\mathrm{SiO}_{2}$ 纳米纤维较低的热导率、 较好的柔性和耐高温性能及纤维气凝胶的多孔结构, 该气凝胶表现出了较好的隔热性能(图 3(b))和压缩 弹性(图 3(c) $)^{[7]}$ 。为进一步降低纤维气凝胶的热导率, 该课题组在纳米纤维气凝胶制备过程中加入了 $\mathrm{SiO}_{2}$ 气凝胶纳米颗粒, 使 $\mathrm{SiO}_{2}$ 气凝胶颗粒均匀分散在纤 维之间的孔隙中, 有效降低了纤维气凝胶热导率, 但其压缩弹性不受影响 ${ }^{[35-36]}$ 。上述纳米纤维气凝胶, 纤维之间均是刚性的陶瓷节点(如硅硼酸盐和 $\mathrm{SiO}_{2}$ ) 连接。这使得其在长期使用过程中弹性降低, 脆性 增大。为解决这一问题, 该组研究者采用硅溶胶作 为纤维之间的节点(图 3(d)), 实现了纤维之间的弹 性连接, 制备的 $\mathrm{SiO}_{2}$ 纳米纤维气凝胶可以经受一百 万次压缩测试而压缩弹性无明显下降, 且能在较宽 的温度范围内 $\left(-196 \sim 1100{ }^{\circ} \mathrm{C}\right)$ 保持优异的压缩弹性 (图 3(e))。该纤维气凝胶最高使用温度可达 $1100{ }^{\circ} \mathrm{C}$, 明显优于其他气凝胶材料(图 3(f) $)^{[37]}$ 。但由于 $\mathrm{SiO}_{2}$ 纤维自身耐高温性能限制 $\left(<1200{ }^{\circ} \mathrm{C}\right), \mathrm{SiO}_{2}$ 纳米纤 维气凝胶不能满足更高温度应用要求。

为进一步提高纤维气凝胶的耐高温性能, $\mathrm{SiC}$ 和莫来石等具有较好耐高温性能的纤维也被用来制 作纤维气凝胶。 $\mathrm{Su}$ 等 ${ }^{[8]}$ 通过气相反应制备了 $\mathrm{SiC}$ 纳 米纤维气凝胶(图 3(g))。由于纤维气凝胶高孔隙率 的特点及 $\mathrm{SiC}$ 纳米纤维自身较好的柔性和耐高温性 能, 该纤维气凝胶表现出了优异的隔热性能(图 3(h)) 和压缩弹性(图 3(i))。最近, Xian 等 ${ }^{[38]}$ 制备了耐高温 性能可达 $1400{ }^{\circ} \mathrm{C}$ 的莫来石纤维气凝胶, 且具有较 

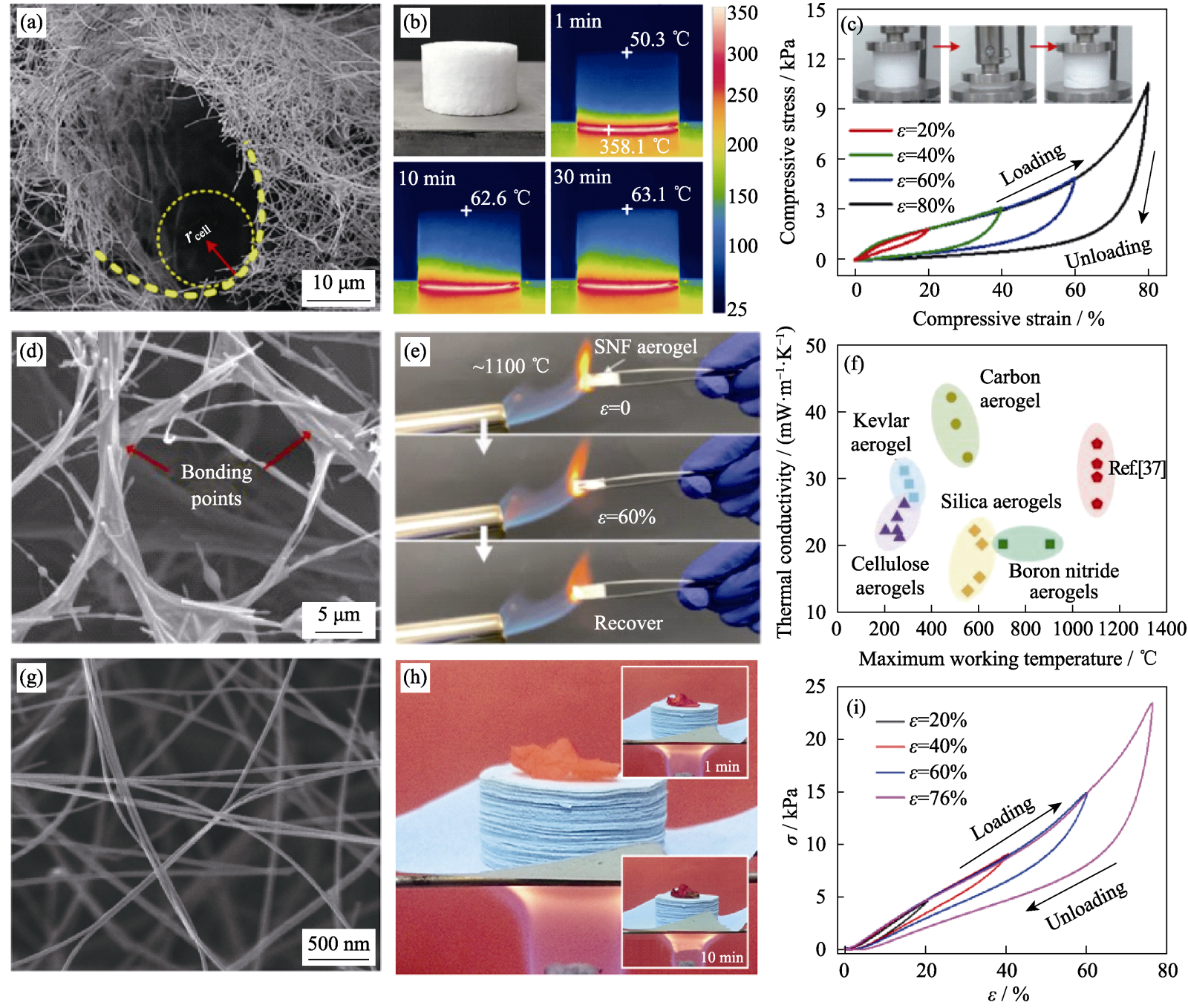

图 $3 \mathrm{SiO}_{2}$ 和 $\mathrm{SiC}$ 纳米纤维气凝胶 ${ }^{[7-8,37]}$

(a)SEM 照片; (b)隔热性能测试红外成像照片; (c) 压缩应力应变图; $\mathrm{SiO}_{2}$ 纳米纤维气凝胶的(d)SEM 照片, (e)高温条件下压缩 性能测试图和(f)热导率对比图; (g)SiC 纳米纤维气凝胶的 SEM 照片, (h) 隔热性能测试光学照片和(i)压缩应力应变曲线

Fig. $3 \quad \mathrm{SiO}_{2}$ and $\mathrm{SiC}$ nanofiber aerogel $\mathrm{l}^{[7-8,37]}$

(a,d) SEM images of $\mathrm{SiO}_{2}$ nanofiber aerogel; (b) Infrared thermal image; (c) Compression stress-strain; (e) Compression test under high temperature; (f) Thermal conductivity comparison; (g) SEM image of SiC nanofiber aerogel; (h) Optical photo of thermal insulation performance of $\mathrm{SiC}$ nanofiber aerogel; (i) Compression stress-strain of $\mathrm{SiC}$ nanofiber aerogel

低的热导率 $\left(0.038 \sim 0.062 \mathrm{~W} \cdot \mathrm{m}^{-1} \cdot \mathrm{K}^{-1}\right)$ 。

此外, 具有较好压缩弹性的碳纳米纤维气凝胶 也得到了广泛研究 ${ }^{[39-41]}$ 。由于碳材料在有氧气氛中, 超过 $450{ }^{\circ} \mathrm{C}$ 的条件下存在明显的氧化, 碳纳米纤维 气凝胶在空气环境中使用温度较低。但其在非氧化 环境中使用温度高达 $2000{ }^{\circ} \mathrm{C}$, 在超高温隔热领域 具有显著优势。

尽管, 目前已制备出了多种微纳陶瓷纤维气凝 胶, 纤维材料的隔热性能有了一定提高。但纤维气 凝胶内部孔洞尺寸较大, 气体热导率较高。减小其 内部孔洞尺寸, 有望进一步提高其隔热性能, 在未 来研究中值得关注。此外, 纤维自身的一些不足(如 强度低和耐高温性能不佳等), 也是限制纤维气凝 胶在高温隔热领域应用的关键问题。

\section{3 中空/多孔微纳陶瓷纤维}

\section{1 中空微纳陶瓷纤维}

在中空纤维中, 固体传热仅能在管壁传输, 因 而中空纤维固体热导率较低。此外, 纤维的空心结 构还对其内部的气体分子具有限制作用。尤其当中 空尺寸小于气体分子的平均自由程时，气体分子的 自由运动被强烈限制, 气体热导率较小 ${ }^{[42]}$ 。Brendel 等 ${ }^{[43]}$ 通过理论计算表明, 在高温条件下, 中空纤维 具有更大的红外辐射线散射截面。这可能是由于中 空结构增加了气固相界面, 红外辐射线通过两相界 面时发生了较多次数的反射、折射和吸收, 辐射传 热减小。中空纤维热传输示意图如图 4 所示。因而, 
Thermal transfer pathways in hollow fiber
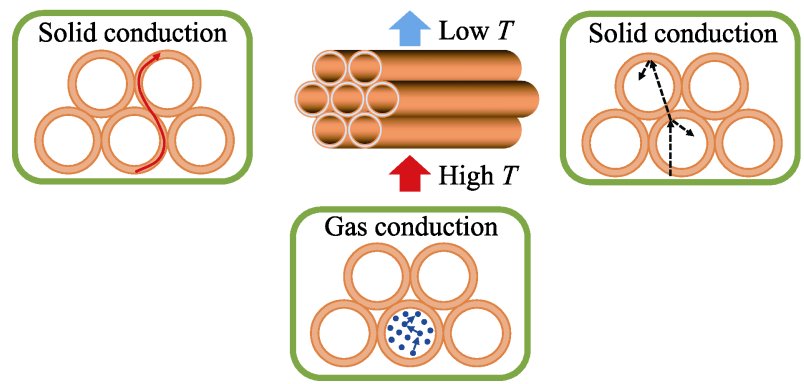

图 4 中空隔热纤维热传导示意图

Fig. 4 Schematic diagram of heat conduction of hollow fiber

相比于实芯纤维, 将纤维设计为中空结构有助于进 一步降低其热导率, 提高纤维材料的隔热性能。目 前, 制备中空微纳陶瓷隔热纤维的方法主要有模板 法和静电纺丝法两种。

模板法制备中空陶瓷隔热纤维具有设备简单、 成本低等优点。南京理工大学王天驰课题组分别采 用蚕丝、木棉、白茅和棉花等植物纤维作为模板制 备了多种直径的中空 $\mathrm{ZrO}_{2}$ 纤维(图 5(a)) ) $^{[44-47]}$ 。相比 传统的实芯 $\mathrm{ZrO}_{2}$ 纤维, 不同温度下中空 $\mathrm{ZrO}_{2}$ 纤维
均具有较低的热导率(图 5(b))。其中, 在 $900{ }^{\circ} \mathrm{C}$ 下 中空 $\mathrm{ZrO}_{2}$ 纤维热导率为 $0.203 \mathrm{~W} \cdot \mathrm{m}^{-1} \cdot \mathrm{K}^{-1}$, 比实芯 纤维热导率下降了约 $13 \%{ }^{[44]}$ 。最近, $\mathrm{Xu}$ 等 ${ }^{[48]}$ 制备了 中空 $\mathrm{Al}_{2} \mathrm{O}_{3}$ 纤维(图 5(c)), 该中空纤维泡沫具有较低 的密度 $\left(0.68 \mathrm{mg} \cdot \mathrm{cm}^{-3}\right)$ 和热导率 $\left(0.022 \mathrm{~W} \cdot \mathrm{m}^{-1} \cdot \mathrm{K}^{-1}\right.$, 图 5(d))。

受北极熊中空结构的毛发具有较好的防寒保温 性能的启发。Zhan 等 ${ }^{[49]}$ 采用直径较细 $(\sim 35 \mathrm{~nm})$, 且 以具有较好分散性的碲纳米线为模板，通过水热反应 结合高温烧成工艺制备了中空碳纳米纤维(图 6(a-b))。 研究发现, 这种中空纳米纤维构成的气凝胶表现出 了较好的压缩弹性(图 6(c)) 和较低的热导率(图 6(d))。 但由于碳材料特性的限制, 其在空气中应用温度较 低 $\left(<450{ }^{\circ} \mathrm{C}\right)$ 。 $\mathrm{Du}$ 等 ${ }^{[50]}$ 制备了中空 $\mathrm{SiO}_{2}$ 纳米纤维, 由中空 $\mathrm{SiO}_{2}$ 纳米纤维构成的气凝胶在室温下具有 较低的热导率 $\left(0.030 \sim 0.033 \mathrm{~W} \cdot \mathrm{m}^{-1} \cdot \mathrm{K}^{-1}\right)$ 。但由于 $\mathrm{SiO}_{2}$ 材料的红外透过性较强, 该纤维气凝胶在高温条件 下辐射热导率较高。

静电纺丝法制备中空纤维具有纤维直径小和形 貌结构易调控等优点, 是制备微纳中空陶瓷纤维的
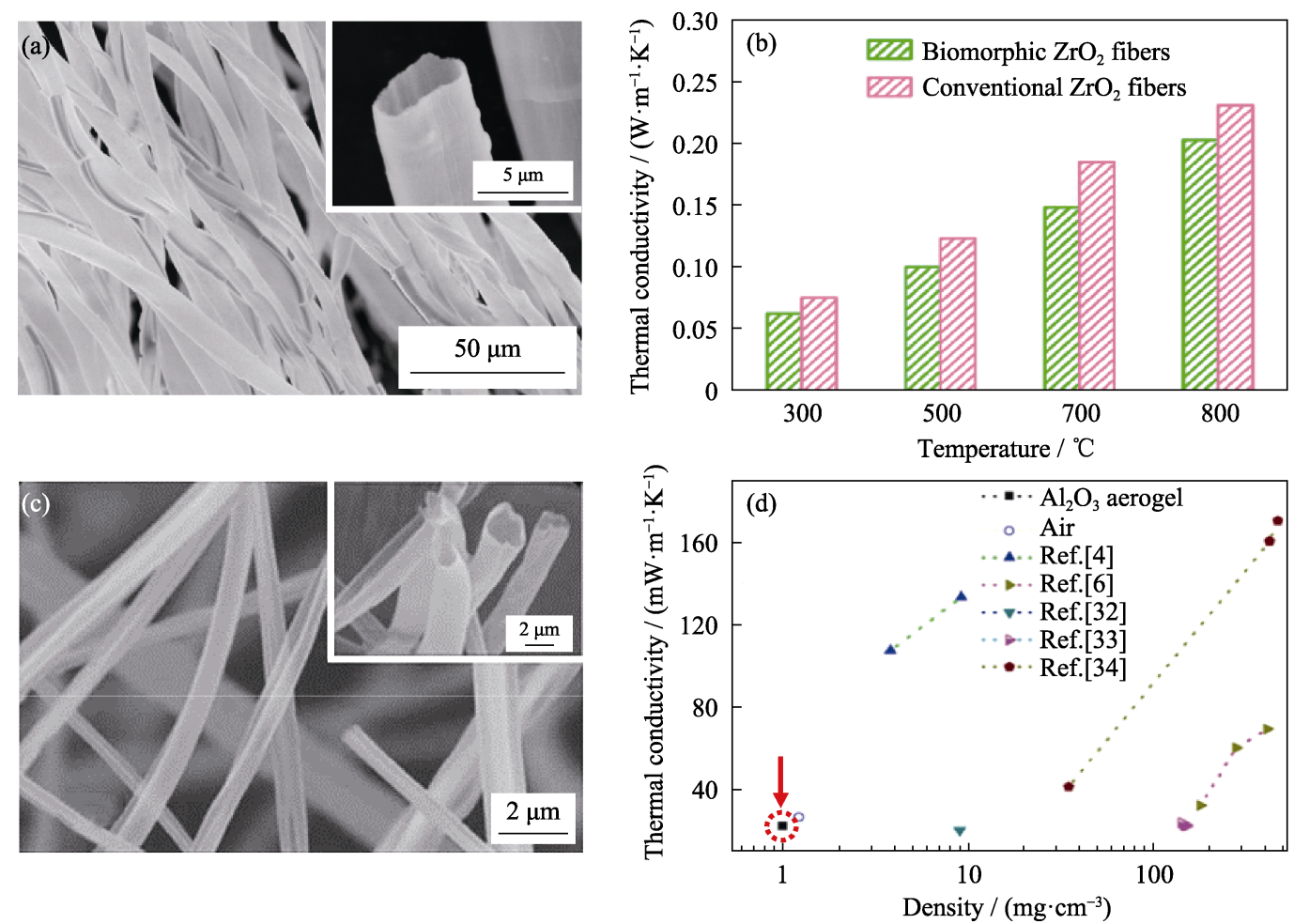

图 5 中空微纳陶瓷纤维 ${ }^{[44,48]}$

(a)中空 $\mathrm{ZrO}_{2}$ 纤维表面和截面 SEM 照片; (b)中空 $\mathrm{ZrO}_{2}$ 纤维与传统实芯 $\mathrm{ZrO}_{2}$ 纤维热导率对比;

(c)中空 $\mathrm{Al}_{2} \mathrm{O}_{3}$ 纤维表面和截面 SEM 照片; (d)中空 $\mathrm{Al}_{2} \mathrm{O}_{3}$ 纤维气凝胶与其他材料热导率对比

Fig. 5 Hollow micro-nano ceramic fiber ${ }^{[44,48]}$

(a) Surface and cross section SEM images of hollow $\mathrm{ZrO}_{2}$ fiber; (b) Comparison of thermal conductivity between hollow $\mathrm{ZrO}_{2}$ fiber and traditional $\mathrm{ZrO}_{2}$ fiber; (c) Surface and cross section SEM images of hollow $\mathrm{Al}_{2} \mathrm{O}_{3}$ fiber;

(d) Thermal conductivity comparison among hollow $\mathrm{Al}_{2} \mathrm{O}_{3}$ fiber aerogel and other materials 
(a)

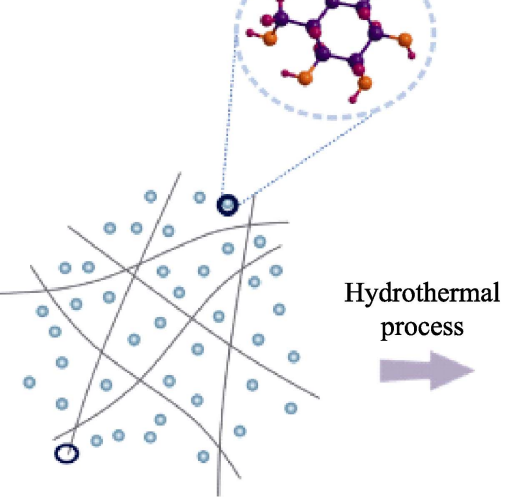

Nanowire

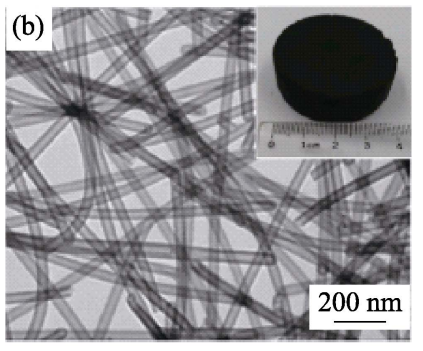

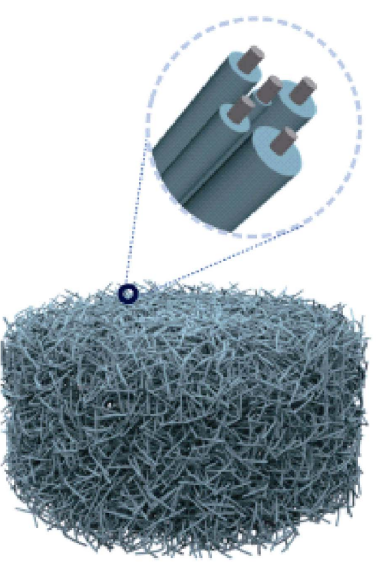

NW@C cable hydrogel

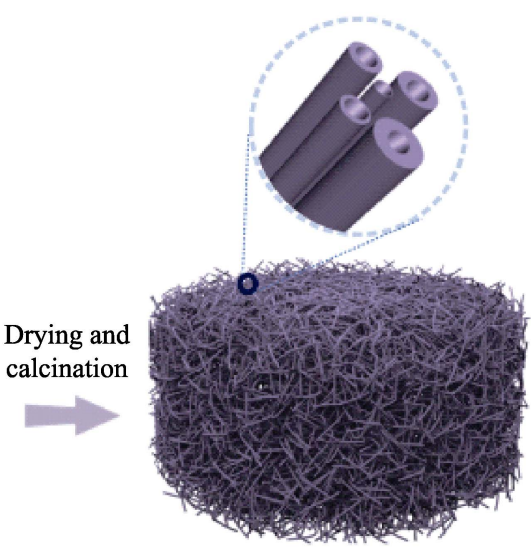

Carbon tube aerogel

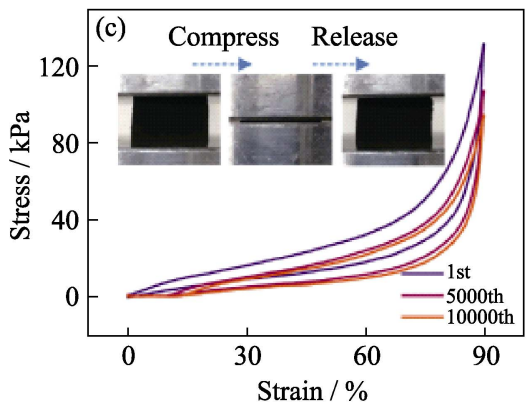

(d)

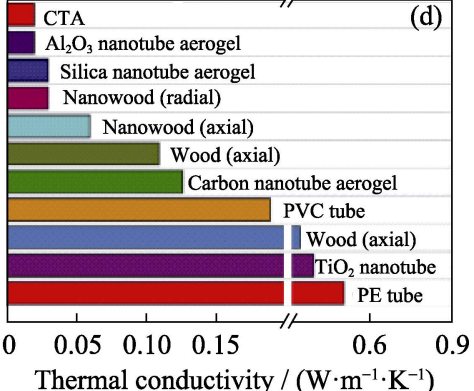

图 6 中空碳纳米纤维气凝胶 ${ }^{[49]}$

(a)制备流程示意图; (b)TEM 照片; (c)中空碳纳米纤维气凝胶经 10000 次压缩应力应变曲线及压缩测试图;

(d)中空碳纳米纤维气凝胶与其他中空材料热导率比较

Fig. 6 Hollow carbon micro-nano fiber aerogel ${ }^{[49]}$

(a) Schematic illustration of the fabrication processes; (b) TEM image; (c) Stress-strain curves for 10000 cycles;

(d) Thermal conductivity comparison among different hollow-structured thermally insulating materials
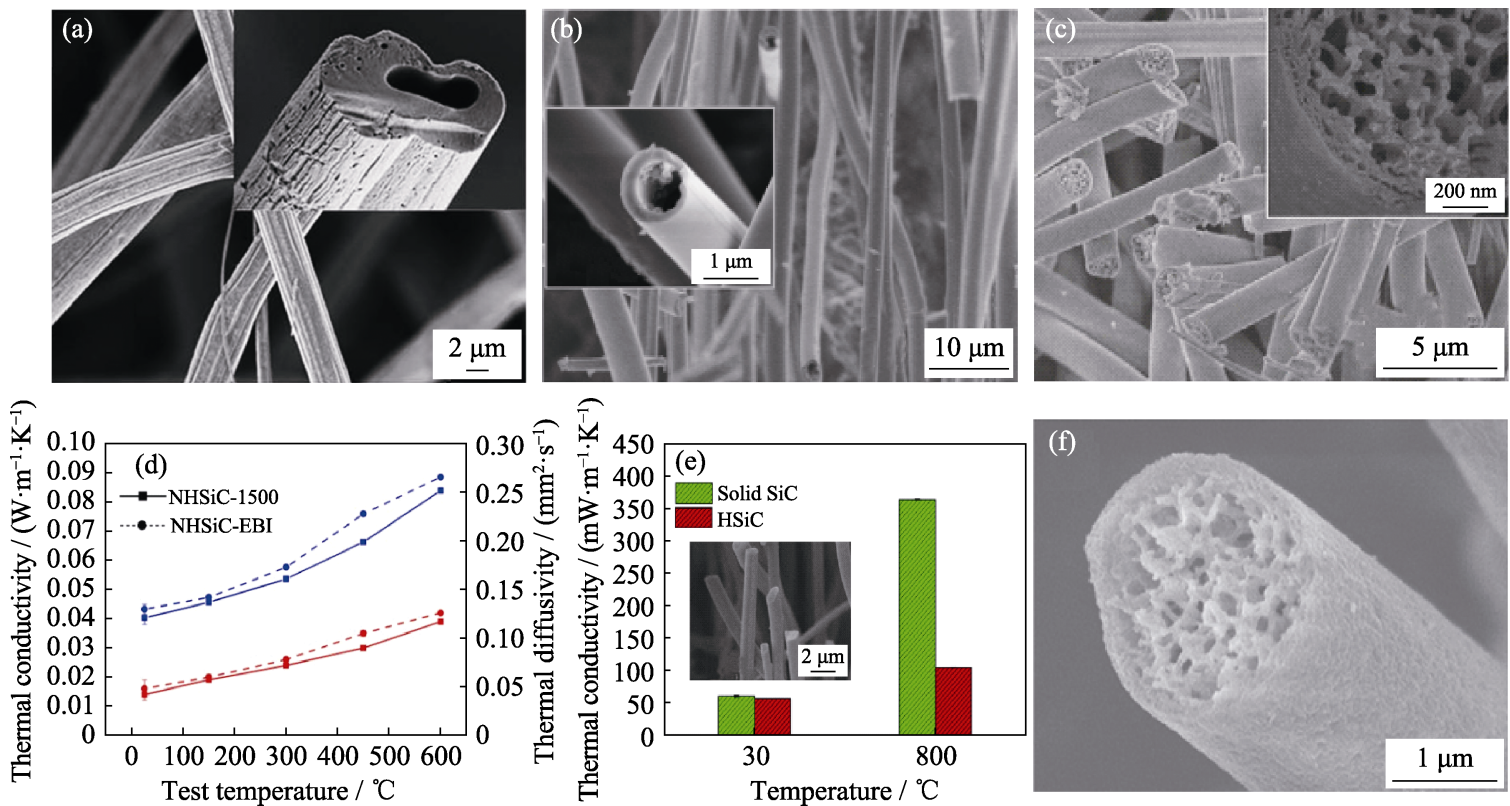

$\widetilde{T} \widetilde{T}$
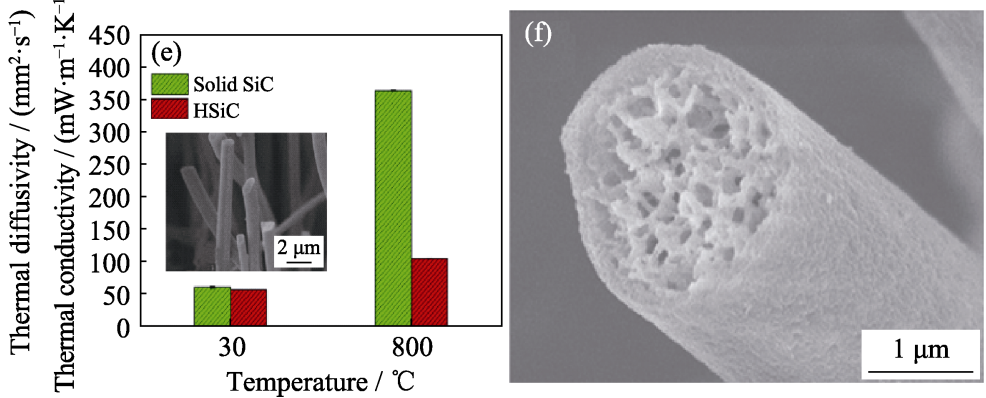

图 7 中空和多空微纳纤维 ${ }^{[51-52,54]}$

(a)氮掺杂中空 $\mathrm{SiC}$ 纤维 SEM 照片; (b)中空 $\mathrm{SiC}$ 纤维 SEM 照片; (c)多孔 $\mathrm{SiO}_{2}-\mathrm{TiO}_{2}$ 纤维 SEM 照片; (d)氮掺杂中空 $\mathrm{SiC}$ 纤维热导率和热扩散系数; (e)中空和实芯 $\mathrm{SiC}$ 纤维热导率; (f)多孔 $\mathrm{SiO}_{2}-\mathrm{ZrO}_{2}$ 纤维 $\mathrm{SEM}$ 照片

Fig. 7 Hollow and porous micro-nano fiber ${ }^{[51-52,54]}$

(a) SEM image of N-doped hollow SiC fiber; (b) SEM image of hollow SiC fiber; (c)SEM image of porous $\mathrm{SiO}_{2}-\mathrm{TiO}_{2}$ fiber;

(d) Thermal conductivities and thermal diffusivities of N-doped hollow SiC fiber; (e) Thermal conductivities of solid $\mathrm{SiC}$ fiber and hollow SiC fiber; (f) $\mathrm{SEM}$ image of $\mathrm{SiO}_{2}-\mathrm{ZrO}_{2}$ fiber 
重要方法。Liu 等 ${ }^{[51]}$ 采用同轴静电纺丝法制备了氮 掺杂的中空 $\mathrm{SiC}$ 纤维(图 7(a))。研究发现, 纤维是由 $\mathrm{SiO}_{x} \mathrm{C}_{y} 、 \mathrm{SiC}_{x} \mathrm{O}_{y} \mathrm{~N}_{z}$ 和自由碳多相组成, 其独特的组成 及中空结构使其表现出了优异的隔热性能, 在 $600{ }^{\circ} \mathrm{C}$ 下热导率仅为 $0.039 \sim 0.042 \mathrm{~W} \cdot \mathrm{m}^{-1} \cdot \mathrm{K}^{-1}$ (图 7(d))。最 近, 本课题组采用微乳液静电纺丝法制备了中空 $\mathrm{SiC}$ 纤维(图 7(b))。研究发现, 中空 $\mathrm{SiC}$ 纤维红外消 光系数明显高于实芯 $\mathrm{SiC}$ 纤维, 在 $800{ }^{\circ} \mathrm{C}$ 下中空 $\mathrm{SiC}$ 纤维热导率为 $0.1049 \mathrm{~W} \cdot \mathrm{m}^{-1} \cdot \mathrm{K}^{-1}$, 比实芯纤维 热导率下降了约 $71 \%$ (图 7(e) $)^{[52]}$ 。

目前, 采用模板法和静电纺丝法已制备出了多 种中空微纳陶瓷隔热纤维, 并取得了较好的隔热效 果。但由于纤维的中空结构极大地增加了纤维的比 表面积, 使得纤维表面存在缺陷的可能性增加。因 而, 目前制备的中空陶瓷纤维强度较低。在未来优 化中空纤维隔热性能的同时, 如何有效提高其强度 也是需关注的重点。

\section{2 多孔微纳陶瓷纤维}

相比于实芯和中空纤维, 多孔结构纤维使得固 体热传输路径更加曲折、对气体分子的限制作用更 大以及对红外辐射线的反射次数更多。因此, 构建 多孔结构微纳陶瓷纤维可进一步降低纤维热导率。 Gbewonyo 等 ${ }^{[53]}$ 制备了多孔碳纳米纤维, 纤维内部 丰富的微孔、介孔和纳米孔结构, 有效降低了其热 导率。与实芯碳纳米纤维膜相比, 多孔碳纳米纤维膜 热导率降低了近 $98 \%$ 。最近, Wang 等 ${ }^{[54]}$ 制备了具有 多级孔结构的 $\mathrm{SiO}_{2}-\mathrm{TiO}_{2}$ 和 $\mathrm{SiO}_{2}-\mathrm{ZrO}_{2}$ 纤维(图 7(c,f))。 纤维内部大量的孔洞, 有助于降低气体和固体热传 导, 并增强对红外辐射线的反射能力, 有望将其应 用于高温隔热领域。

最近, 研究人员制备了具有纳米多孔结构的有 机气凝胶纤维, 纤维内部丰富的纳米孔结构使其展 现出了优异的隔热性能 ${ }^{[55-57]}$ 。受此启发, 进一步调 控多孔陶瓷纤维的孔结构, 制备纳米多孔结构的 “微纳陶瓷气凝胶纤维”有望显著降低陶瓷纤维热导 率, 是未来微纳陶瓷隔热纤维发展的重要方向。但 目前尚未有“陶瓷气凝胶纤维”的报道, 这主要是由 于其制备困难, 且纤维内部大量的纳米孔结构会对 纤维力学性能产生不利影响。

目前, 多孔微纳陶瓷隔热纤维正处于新兴阶段, 制备具有较好力学性能的多孔微纳陶瓷隔热纤维还 存在着较大挑战, 是其未来发展中面临的难题。此 外, 孔结构与隔热性能之间的构效关系等科学问题 还有待进一步研究。且对于多孔纤维的隔热机制研 究还不充分, 在未来研究中也需重点关注。

\section{4 复合微纳陶瓷纤维}

由普朗克定律可知, 随物体温度升高, 其光谱 辐射强度逐渐增强, 辐射传热占比增大。在高温条 件下 $\left(T>1000{ }^{\circ} \mathrm{C}\right)$, 黑体辐射波长主要集中在 $1 \sim$ $8 \mu \mathrm{m}$ 的近红外以及中红外波段 ${ }^{[58-59]}$ 。因而, 理想的 高温隔热材料需在此波段内具有较低的红外透过性。 但对于绝大多数氧化物陶瓷纤维 $\left(\mathrm{ZrO}_{2} 、 \mathrm{SiO}_{2}\right.$ 和 $\mathrm{Al}_{2} \mathrm{O}_{3}$ 等), 其在 $1 \sim 8 \mu \mathrm{m}$ 波段内红外透过率较高 ${ }^{[43,60-61]}$ 。 因而, 其在高温条件下的热导率较高。如何有效降 低氧化物陶瓷纤维的红外透过性, 是提高其高温隔 热性能的关键。

当红外辐射线(总能量 $E_{\mathrm{L}}$ )传递到纤维表面时会 发生反射(反射能量 $E_{\mathrm{R}}$ )、吸收(吸收能量 $E_{\mathrm{A}}$ ) 和透过 (透过能量 $E_{\mathrm{T}}$ ), 如图 8 所示。由能量守恒定律可知, 只有增加对红外辐射线的反射和吸收才能降低透过 的红外辐射能量。目前, 降低纤维的红外透过性主 要有两种方法: 1)在纤维表面构筑高反射率涂层, 提高纤维对红外辐射线的反射能力; 2)在纤维中复 合具有较高红外反射和吸收能力的遮光剂相。

\section{1 高反射涂层复合微纳陶瓷纤维}

目前, 在纤维表面构筑高反射率的涂层种类主

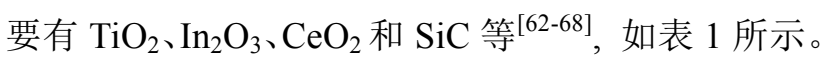
Hass 等 ${ }^{[64]}$ 在 $\mathrm{Al}_{2} \mathrm{O}_{3}$ 纤维表面分别制备了 $\mathrm{TiO}_{2}$ 、 $\mathrm{TiO}_{2} / \mathrm{SiO}_{2} / \mathrm{TiO}_{2}$ 和 $\mathrm{TiO}_{2}-\mathrm{Pt}$ 纳米颗粒复合薄膜。结果 表明, 在纤维表面构筑 $\mathrm{TiO}_{2} / \mathrm{SiO}_{2} / \mathrm{TiO}_{2}$ 多层反射膜, 具有较好的红外反射性能, 有效降低了红外透过 性。与无涂层纤维相比, 其消光系数提高了近 $40 \%$ 。 此外, 在 $\mathrm{SiO}_{2}$ 纤维表面制备掺锡氧化铟(ITO)反射膜 和 ITO/Ag/ITO 多层反射膜也可有效降低纤维的红 外透过率 ${ }^{[59,65]}$ 。Gan 等 ${ }^{[66]}$ 采用水热反应法在 $\mathrm{ZrO}_{2}$ 纤 维表面制备了具有较高反射率的 $\mathrm{CeO}_{2}$ 涂层(图 9(a))。

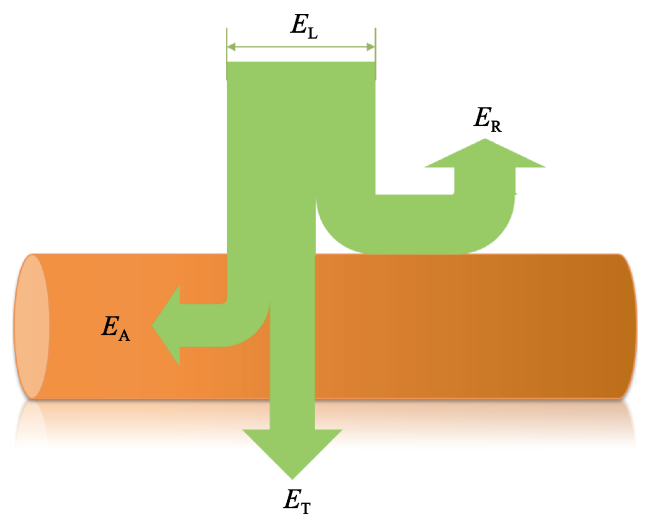

图 8 红外辐射线在纤维中传输示意图

Fig. 8 Schematic of infrared radiation transmission in the fiber 
表 1 高反射率涂层纤维制备方法及涂层种类

Table 1 Preparation method and coating types of high-reflectivity coated fiber

\begin{tabular}{ccccc}
\hline Fiber & Method & Infrared reflectance layer & Coating thickness/ $\mu$ m & Ref. \\
\hline $\mathrm{Al}_{2} \mathrm{O}_{3}$ & Dip-coating & $\mathrm{TiO}_{2}, \mathrm{TiO}_{2} / \mathrm{SiO}_{2} / \mathrm{TiO}_{2}, \mathrm{TiO}_{2}-\mathrm{Pt}$ & - & {$[64]$} \\
$\mathrm{SiO}_{2}$ & Dip-coating & $\mathrm{ITO}, \mathrm{ITO} / \mathrm{Ag} / \mathrm{ITO}$ & $\sim 0.2$ & {$[60,65]$} \\
$\mathrm{ZrO}_{2}$ & Hydrothermal & $\mathrm{CeO}_{2}$ & $52-214$ & {$[66]$} \\
$\mathrm{Mullite}$ & Hydrothermal & $\mathrm{TiO}_{2}$ & - & {$[67]$} \\
$\mathrm{ZrO}_{2}$ & Hydrothermal & $\mathrm{TiO}_{2}$ & $89-236$ & {$[68]$} \\
$\mathrm{Mullite}$ & Dip-coating & $\mathrm{SiC}$ & $\sim 0.8$ & {$[69]$} \\
\hline
\end{tabular}
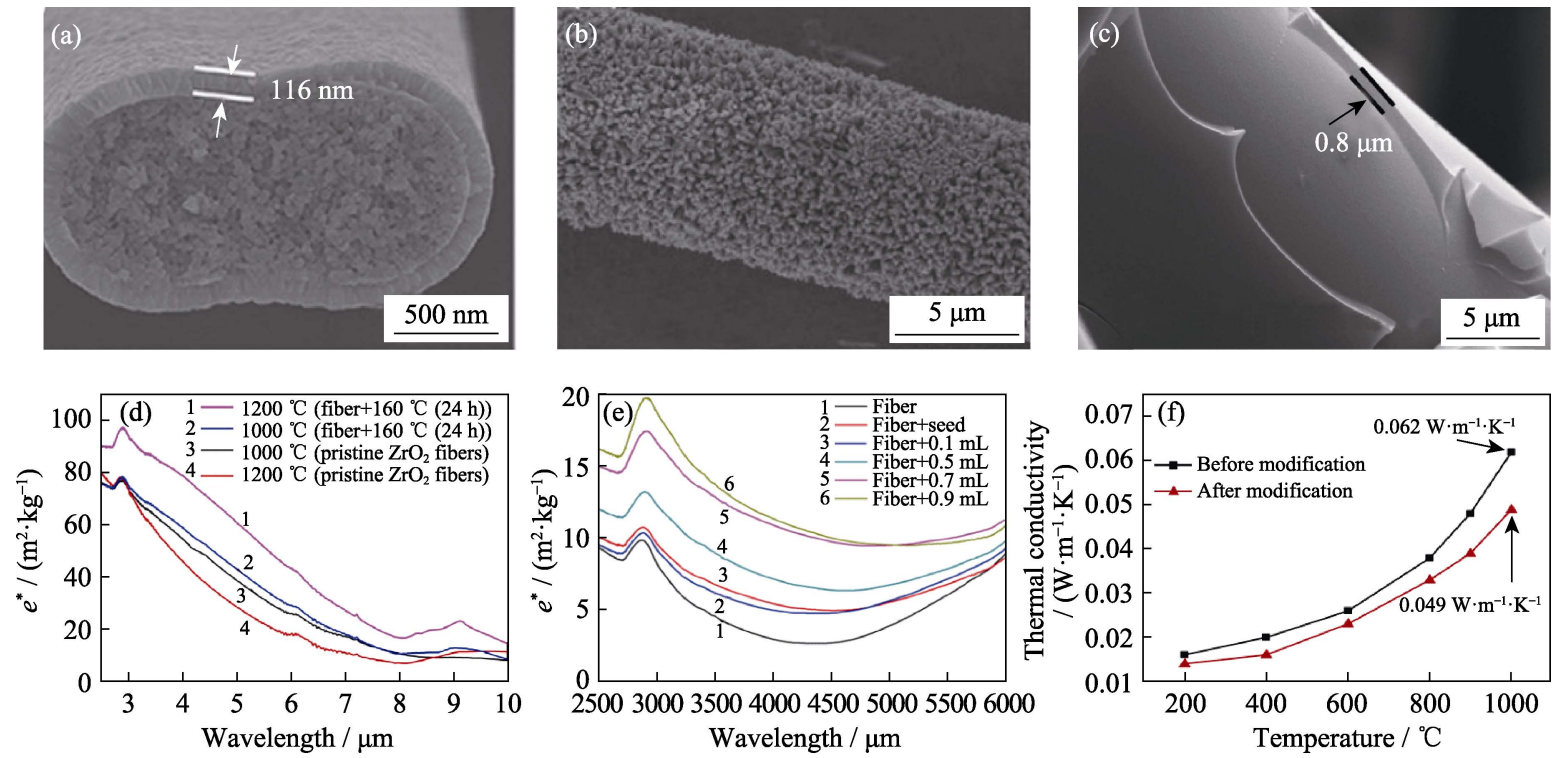

图 9 高反射率涂层纤维 ${ }^{[66-67,69]}$

(a) $\mathrm{CeO}_{2} / \mathrm{ZrO}_{2}$ 纤维 $\mathrm{SEM}$ 照片; (b) $\mathrm{TiO}_{2}$ /莫来石纤维 $\mathrm{SEM}$ 照片; (c)SiC/莫来石纤维表面 $\mathrm{SEM}$ 照片; (d) $\mathrm{ZrO}_{2}$ 纤维和 $\mathrm{CeO}_{2} / \mathrm{ZrO}_{2}$ 纤维的比消光系数对比; (e)莫来石纤维和 $\mathrm{TiO}_{2}$ / 莫来石纤维比消光系数对比; (f)莫来石纤维和 $\mathrm{SiC} /$ 莫来石纤维增强气凝胶复合材料热导率对比

Fig. 9 High-reflectivity coated fiber ${ }^{[66-67,69]}$

(a) SEM image of $\mathrm{ZrO}_{2}$ fiber with $\mathrm{CeO}_{2}$ coating; (b) SEM image of mullite fiber with $\mathrm{TiO}_{2}$ coating; (c) SEM image of mullite fiber with $\mathrm{SiC}$ coating; (d) Specific extinction coefficients comparison of $\mathrm{ZrO}_{2}$ fiber and $\mathrm{CeO}_{2} / \mathrm{ZrO}_{2}$ fiber;

(e) Specific extinction coefficients comparison of mullite fiber and $\mathrm{TiO}_{2} /$ mullite fiber; (f) Thermal conductivity comparison of mullite fiber and $\mathrm{SiC} /$ mullite fiber reinforced aerogel composite

结果表明, 在 $2.5 \sim 10 \mu \mathrm{m}$ 波长范围内, 涂层纤维平 均红外消光系数比无涂层纤维提高了 1 倍(图 9(d))。 Yang 等 ${ }^{[67]}$ 采用溶剂热法在莫来石纤维表面制备了 反射率更高的 $\mathrm{TiO}_{2}$ 纳米棒涂层(图 9(b))。结果表明, 在 3 6 $\mu \mathrm{m}$ 的波长范围内, 涂层纤维有效消光系数 比无涂层纤维提高了 2 4 倍(图 9(e))。最近, Ma 等 ${ }^{[68]}$ 在 $\mathrm{ZrO}_{2}$ 纤维表面制备了 $\mathrm{TiO}_{2}$ 涂层。虽然, $\mathrm{ZrO}_{2}$ 纤维 表面制备 $\mathrm{TiO}_{2}$ 涂层提高了对红外辐射线的反射能 力, 但由于 $\mathrm{TiO}_{2}$ 自身较高的固体热导率使纤维之间 固体热传导增强, 反而导致涂层纤维热导率比无涂 层纤维更高。

$\mathrm{Xu}$ 等 ${ }^{[69]}$ 在莫来石纤维表面制备了 $\mathrm{SiC}$ 涂层 (图 9(c))。研究发现, 莫来石纤维表面的 $\mathrm{SiC}$ 涂层使得 纤维红外消光系数增加。由纤维增强的 $\mathrm{Al}_{2} \mathrm{O}_{3}-\mathrm{SiO}_{2}$
气凝胶复合材料热导率测试结果可以看出, 涂层纤 维制备的气凝胶复合材料热导率较低(图 9(f))。由于 $\mathrm{SiC}$ 自身固体热导率较高, 使得纤维固体热传导增 加, 因而制备涂层后纤维隔热性能提高并不明显。

目前，通过在纤维表面构筑高反射涂层，使得 氧化物陶瓷纤维的红外遮蔽性能有了一定提高。但 由于部分高反射率材料, 其自身固体热导率较高 (如 $\mathrm{TiO}_{2}$ 和 $\mathrm{SiC}$ 等)。将其作为红外反射涂层后，会 使纤维之间的固体热传导增加, 综合热导率降低不 明显或反而增大。因此，在选择反射涂层时也需考 虑其固体热导率，尽量选用固体热导率较低但反射 率较高的材料作为反射涂层。此外, 由于涂层与纤 维之间结合较弱, 在长期使用过程中涂层易脱落, 这也是未来涂层纤维发展需解决的问题。 


\section{2 复相微纳陶瓷纤维}

除了在纤维表面构筑高反射涂层外，将具有低 固体热导率和高红外遮蔽性能的材料复合, 设计复 相微纳陶瓷纤维, 有望制备出兼具低固体热导率和 高红外遮蔽性能的新型微纳陶瓷隔热纤维。目前, 制备的复相微纳陶瓷纤维主要有两种, 两相复合纤 维和多元均相复合纤维。前期, 本课题组 ${ }^{[70]}$ 通过控 制熟化工艺, 制备了表面富 $\mathrm{ZrO}_{2}$ 层的 $\mathrm{ZrO}_{2} / \mathrm{SiC}$ 两相 复合纤维(图 $10(a, b))$ 。纤维表面的 $\mathrm{ZrO}_{2}$ 层具有较低 的固体热导率, 有助于降低纤维之间固体热传导。 同时, 芯部的 $\mathrm{SiC}$ 具有较好的红外遮蔽性能, 能有 效降低辐射热传输。制备的 $\mathrm{ZrO}_{2} / \mathrm{SiC}$ 复相纤维是较 理想的高温隔热材料 ${ }^{[70]}$ 。近期, 本课题组设计
制备了多元复相 SiZrOC 纳米纤维(图 10(c) ) ${ }^{[14]}$ 。研 究发现, 纤维是由 $\mathrm{SiC} 、 \mathrm{SiO}_{2}$ 、自由碳和 $\mathrm{ZrO}_{2}$ 多相 组成。纤维内部的复杂多相结构使其内部存在大量 相界面, 增加了声子-界面散射, 纤维固体热导率较 低。同时, 纤维内部的 $\mathrm{SiC}$ 和自由碳具有较高的红 外反射和吸收能力, 有效降低了辐射热传导, 因而 制备的 SiZrOC 纤维在高温条件下具有较低的热导 率(图 10(d)), 纤维隔热机理示意图如图 10(e)所示。 因此, 设计复相微纳陶瓷隔热纤维有望制备出高效 隔热纤维, 这也是未来微纳陶瓷隔热纤维发展的重 要方向之一。

通过在纤维表面构筑高反射涂层或在纤维内部 复合遮光剂相, 有效提高了微纳陶瓷纤维的高温隔 (a)

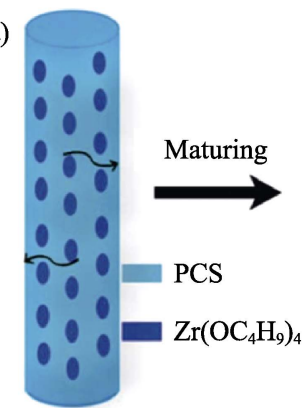

As-spun fiber

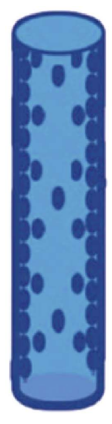

Maturated fiber

Curing \& Pyrolysis

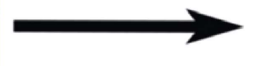

$\mathrm{ZrO}_{2}$

$\mathrm{SiC}$

Gradient $\mathrm{ZrO}_{2} / \mathrm{SiC}$ fiber
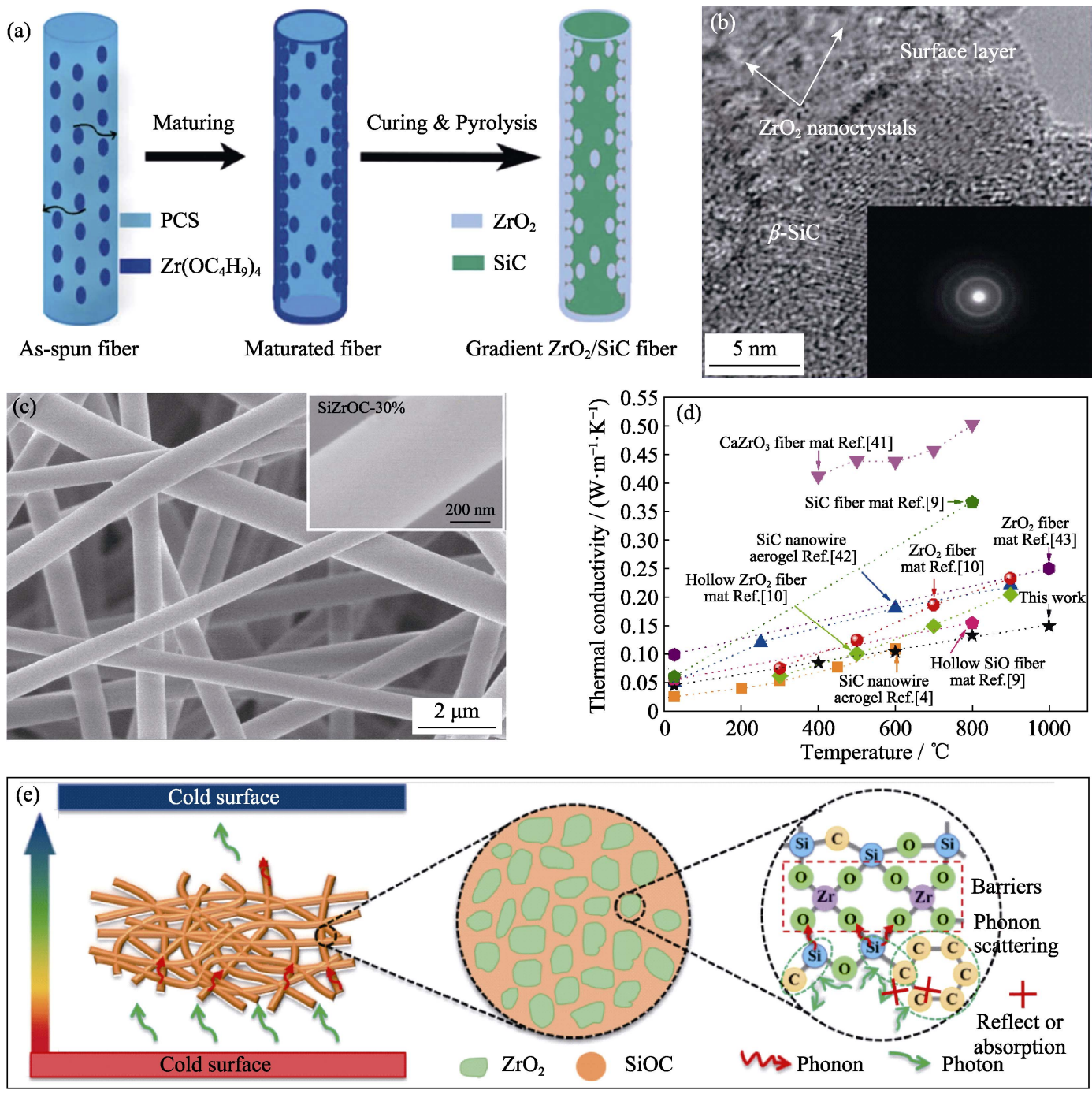

图 10 复相微纳陶瓷纤维 ${ }^{[14,70]}$

(a) $\mathrm{ZrO}_{2} / \mathrm{SiC}$ 纤维制备示意图; (b) $\mathrm{ZrO}_{2} / \mathrm{SiC}$ 纤维 TEM 照片; (c) $\mathrm{SiZrOC}$ 纤维 $\mathrm{SEM}$ 照片;

(d) SiZrOC 纤维热导率对比; (e) SiZrOC 纤维隔热机理示意图

Fig. 10 Composite micro-nano ceramic fiber ${ }^{[14,70]}$

(a) Schematic illustration of the preparation of $\mathrm{ZrO}_{2} / \mathrm{SiC}$ fiber; (b) TEM images of $\mathrm{ZrO}_{2} / \mathrm{SiC}$ fiber; (c) SEM images of SiZrOC fiber; (d) Thermal conductivity comparison of SiZrOC fiber with other ceramic fibers;

(e) Schematic illustration of thermal insulation mechanisms of SiZrOC fibers 
热性能。相比于传统陶瓷纤维和气凝胶隔热材料, 复合微纳陶瓷纤维较佳的力学性能和优异的红外遮 蔽性能，使其在高温隔热领域具有显著优势。

\section{5 总结及展望}

微纳陶瓷隔热纤维以其较佳的耐高温和隔热性 能，在高温隔热领域具有广阔的应用前景。通过优化 纤维的组成和结构可进一步改善其隔热性能, 是当 前微纳陶瓷隔热纤维研究的重点。尽管目前微纳 陶瓷纤维的隔热性能有了一定提高, 但仍面临诸多 挑战:

1)目前制备的微纳陶瓷隔热纤维强度相对较 低, 尤其是对于中空和多孔结构纤维。如何有效提高 纤维的强度是未来微纳陶瓷隔热纤维发展需关注的 重点。

2)对于广泛应用的氧化物陶瓷纤维，其在高温 条件下晶粒长大, 致使纤维易脆, 且强度下降显著。 因此，在优化纤维隔热性能的同时，提高其耐高温 性能也是未来必须解决的问题。

3)多孔微纳陶瓷隔热纤维处于新兴阶段，结合 理论计算和实验手段，阐明其高温隔热机制，对于 指导纤维的结构设计和提高其隔热性能具有重要的 理论指导意义。

4)目前，制备微纳陶瓷隔热纤维生产效率较低, 难以满足实际工业应用需求。开发高效的微纳陶瓷 隔热纤维规模化生产方法是推动其在隔热领域广泛 应用的关键问题。

\section{参考文献:}

[1] CHEN Y F, HONG C Q, HU C L, et al. Ceramic-based thermal protection materials for aerospace vehicle. Advanced Ceramics, 2017, 38(5): 311-390.

[2] BEHRENS B, MULLER M. Technologies for thermal protection systems applied on reusable launcher. Acta Astronautica, 2004, 55(3-9): 529-536

[3] WANG C A, LANG Y, HU L F, et al. Research progress on lightweight and high strength heat-insulating porous ceramics. Journal of Ceramics, 2017, 38(6): 287-296.

[4] TERESA L, MARIA T P A, LUISA D. Silica aerogel composites with embedded fibres: a review on their preparation, properties and applications. Journal of Materials Chemistry A, 2019, 7: 22768-22802.

[5] LUO Y, JIANG Y G, FENG J Z, et al. Progress on the preparation of $\mathrm{SiO}_{2}$ aerogel composites by ambient pressure drying technique. Materials Review, 2018, 32(5): 780-787.

[6] XU X, ZHANG Q, HAO M, et al. Double-negative-index ceramic aerogels for thermal superinsulation. Science, 2019, 363(6428): 723-727

[7] SI Y, WANG X, DOU L, et al. Ultralight and fire-resistant ceramic nanofibrous aerogels with temperature-invariant superelasticity. Science Advances, 2018, 4(4): eaas8925.

[8] SU L, WANG H, NIU M, et al. Ultralight, recoverable, and high-temperature-resistant SiC nanowire aerogel. ACS Nano, 2018,
12(4): 3103-3111.

[9] SABETZADEH N, BAHRAMBEYGI H, RABBI A, et al. Thermal conductivity of polyacrylonitrile nanofibre web in various nanofibre diameters and surface densities. Micro \& Nano Letters, 2012, 7(7): 662-666

[10] YAN J, HAN Y, XIA S, et al. Polymer template synthesis of flexible $\mathrm{BaTiO}_{3}$ Crystal nanofibers. Advanced Functional Materials, 2019, 29(51): 1907919.

[11] YAN J, ZHAO Y, WANG X, et al. Polymer template synthesis of soft, light, and robust oxide ceramic films. iScience, 2019, 15: 185-195.

[12] ARAMBAKAM R, TAFRESHI H V, POURDEYHIMI B. A simple simulation method for designing fibrous insulation materials. Materials \& Design, 2013, 44: 99-106.

[13] ARAMBAKAM R, TAFRESHI H V, POURDEYHIMI B. Dual-scale 3-D approach for modeling radiative heat transfer in fibrous insulations. International Journal of Heat and Mass Transfer, 2013, 64: 1109-1117.

[14] ZHANG X S, WANG B, WU N, et al. Flexible and thermal-stable SiZrOC nanofiber membranes with low thermal conductivity at high-temperature. Journal of the European Ceramic Society, 2020, 40(5): $1877-1885$

[15] DARYABEUGi K, CUNNINGTON G R, KNUTSON J R. Heat transfer modeling for rigid high-temperature fibrous insulation. Journal of Thermophysics and Heat Transfer, 2013, 27(3): 414-421.

[16] HU F, WU S, SUN Y. Hollow structured materials for thermal insulation. Advanced Materials, 2019, 31(38): 1801001.

[17] MACHADO H A. Modeling heat transfer with micro-scale natural convection in fibrous insulation. Journal of the Brazilian Society of Mechanical Sciences and Engineering, 2014, 36(4): 847-857.

[18] DARYABEUGI K, CUNNINGTON G R, KNUTSON J R. Combined heat transfer in high-porosity high-temperature fibrous insulation: theory and experimental validation. Journal of Thermophysics and Heat Transfer, 2011, 25(4): 536-546.

[19] SHIN S, WANG Q, LUO J, et al. Advanced materials for hightemperature thermal transport. Advanced Functional Materials, 2020, 30: 1904815 .

[20] GIBSON P W, LEE C, KO F, et al. Application of nanofiber technology to nonwoven thermal insulation. Journal of Engineered Fibers and Fabrics, 2007, 2(2): 32-40.

[21] WANG B, WANG Y D. Effect of fiber diameter on thermal conductivity of the electrospun carbon nanofiber mats. Advanced Materials Research, 2011, 332: 672-677.

[22] YAN J, ZHANG Y, ZHAO Y, et al. Transformation of oxide ceramic textiles from insulation to conduction at room temperature. Science Advances, 2020, 6(6): eaay8538.

[23] ZHU W, GUO A, XUE Y, et al. Mechanical evaluations of mullite fibrous ceramics processed by filtration and in situ pyrolysis of organic precursor. Journal of the European Ceramic Society, 2019, 39(4): 1329-1335.

[24] HE F, LI W, ZKOU L, et al. Preparation and characterization of the three-dimensional network mullite porous fibrous materials by pressure and freeze-casting method. Ceramics International, 2019, 45(3): 3954-3960.

[25] XUE J, WU T, DAI Y, et al. Electrospinning and electrospun nanofibers: methods, materials, and applications. Chemical Reviews, 2019, 119(8): 5298-5415.

[26] WU N, WANG B, WANG Y D. Enhanced mechanical properties of amorphous SiOC nanofibrous membrane through in situ embedding nanoparticles. Journal of the American Ceramic Society, 2018, 101(10): 4763-4772.

[27] SI Y, MAO X, ZHENG H, et al. Silica nanofibrous membranes with ultra-softness and enhanced tensile strength for thermal insulation. RSC Advances, 2015, 5(8): 6027-6032.

[28] MAO X, BAI Y, YU J, et al. Flexible and highly temperature resistant polynanocrystalline zirconia nanofibrous membranes designed for air filtration. Journal of the American Ceramic Society, 2016, 99(8): 2760-2768.

[29] ZHANG P, CHEN D, JIAO X. Fabrication of flexible $\alpha$-alumina 
fibers composed of nanosheets. European Journal of Inorganic Chemistry, 2012, 2012(26): 4167-4173

[30] LI W, ZHAO X M, WANG Y F, et al. Fabrication and mechanical properties of flexible gamma- $\mathrm{Al}_{2} \mathrm{O}_{3}$ nanofibrous membranes. Chemical Journal of Chinese Universities, 2017, 38(6): 915-921.

[31] YUAN K, WANG X, LIU H, et al. Formation of barium zirconate fibers for high-temperature thermal insulation applications. Journal of the American Ceramic Society, 2016, 99(9): 2913-2919.

[32] SHI S, YUAN K, XU C, et al. Electrospun fabrication, excellent high-temperature thermal insulation and alkali resistance performance of calcium zirconate fiber. Ceramics International, 2018, 44(12): 14013-14019.

[33] XIE Y, WANG L, LIU B, et al. Flexible, controllable, and high-strength near-infrared reflective $\mathrm{Y}_{2} \mathrm{O}_{3}$ nanofiber membrane by electrospinning a polyacetylacetone-yttrium precursor. Materials \& Design, 2018, 160: 918-925.

[34] SI Y, YU J, TANG X, et al. Ultralight nanofibre-assembled cellular aerogels with superelasticity and multifunctionality. Nature Communications, 2014, 5(1): 1-9.

[35] DOU L, CHENG X, ZHANG X, et al. Temperature-invariant superelastic, fatigue resistant, and binary-network structured silica nanofibrous aerogels for thermal superinsulation. Journal of Materials Chemistry A, 2020, 32(2): 1904331.

[36] DOU L, ZHANG X, CHENG X, et al. Hierarchical cellular structured ceramic nanofibrous aerogels with temperature-invariant superelasticity for thermal insulation. ACS Applied Materials \& Interfaces, 2019, 11(32): 29056-29064.

[37] WANG F, DOU L, DAI J, et al. In situ synthesis of biomimetic silica nanofibrous aerogels with temperature-invariant superelasticity over one million compressions. Angewandte Chemie International Edition, 2020, 59(21): 8285-8292.

[38] XIAN L, ZHANG Y, WU Y, et al. Microstructural evolution of mullite nanofibrous aerogels with different ice crystal growth inhibitors. Ceramics International, 2020, 46(2): 1869-1875.

[39] YU Z L, QIN B, MA Z Y, et al. Superelastic hard carbon nanofiber aerogels. Advanced Materials, 2019, 31(23): 1900651.

[40] LI C, DING Y W, HU B C, et al. Temperature-invariant superelastic and fatigue resistant carbon nanofiber aerogels. Advanced Materials, 2020, 32(2): 1904331.

[41] ZHANG J, LI B, LI L, et al. Ultralight, compressible and multifunctional carbon aerogels based on natural tubular cellulose. Journal of Materials Chemistry A, 2016, 4(6): 2069-2074.

[42] RUCKDESCHEL P, PHILIPP A, RETSCH M. Understanding thermal insulation in porous, particulate materials. Advanced Functional Materials, 2017, 27(38): 1702256.

[43] BRENDEL H, SEIFERT G, RARTHER F. Heat transfer properties of hollow-fiber insulation materials at high temperatures. Journal of Thermophysics and Heat Transfer, 2017, 31(2): 463-472.

[44] WANG T C, ZHANG Z, DAI C, et al. Amorphous silicon and silicates-stabilized $\mathrm{ZrO}_{2}$ hollow fiber with low thermal conductivity and high phase stability derived from a cogon template. Ceramics International, 2019, 45(6): 7120-7126.

[45] WANG T C, KONG S, CHANG L, et al. Preparation and heat-insulating property of the bio-inspired $\mathrm{ZrO}_{2}$ fibers based on the silk template. Ceramics International, 2012, 38(8): 6783-6788.

[46] WANG T C, YU Q, KONG J, et al. Synthesis and heat-insulating properties of yttria-stabilized $\mathrm{ZrO}_{2}$ hollow fibers derived from a ceiba template. Ceramics International, 2017, 43(12): 9296-9302.

[47] WANG T C, YU Q, KONG J. Preparation and heat-insulating properties of biomorphic $\mathrm{ZrO}_{2}$ hollow fibers derived from a cotton template. International Journal of Applied Ceramic Technology, 2018, 15(2): 472-478.

[48] XU C, WANG $\mathrm{H}$, SONG $\mathrm{J}$, et al. Ultralight and resilient $\mathrm{Al}_{2} \mathrm{O}_{3}$ nanotube aerogels with low thermal conductivity. Journal of the American Ceramic Society, 2018, 101(4): 1677-1683.

[49] ZHAN H J, WU K J, HU Y L, et al. Biomimetic carbon tube aerogel enables super-elasticity and thermal insulation. Chem,
2019, 5(7): 1871-1882.

[50] DU A, WANG H, ZHOU B, et al. Multifunctional silica nanotube aerogels inspired by polar bear hair for light management and thermal insulation. Chemistry of Materials, 2018, 30(19): 6849-6857.

[51] LIU Y, LIU Y, CHOI W C, et al. Highly flexible, erosion resistant and nitrogen doped hollow $\mathrm{SiC}$ fibrous mats for high temperature thermal insulators. Journal of Materials Chemistry A, 2017, 5(6): 2664-2672.

[52] TIAN Q, WU N, WANG B, et al. Fabrication of hollow SiC ultrafine fibers by single-nozzle electrospinning for high-temperature thermal insulation application. Materials Letters, 2019, 239: 109-112.

[53] GBEWONYO S, CARPENTER A W, GAUSE C B, et al. Low thermal conductivity carbon fibrous composite nanomaterial enabled by multi-scale porous structure. Materials \& Design, 2017, 134: 218-225.

[54] WANG Y D, HUANG H, ZHAO Y, et al. Self-assembly of ultralight and compressible inorganic sponges with hierarchical porosity by electrospinning. Ceramics International, 2020, 46(1): 768-774.

[55] LIU Z, LYU J, FANG D, et al. Nanofibrous Kevlar aerogel threads for thermal insulation in harsh environments. ACS Nano, 2019, 13(5): 5703-5711.

[56] ZHOU J, HSIEH Y L. Nanocellulose aerogel-based porous coaxial fibers for thermal insulation. Nano Energy, 2020, 68: 104305.

[57] YANG H, WANG Z, LIU Z, et al. Continuous, strong, porous silk firoin-based aerogel fibers toward textile thermal insulation. Polymers, 2019, 11(11): 1899.

[58] YANG J, WU H, WANG M, et al. Prediction and optimization of radiative thermal properties of ultrafine fibrous insulations. Applied Thermal Engineering, 2016, 104: 394-402.

[59] YANG L L, GE D, WEI H, et al. Morphology and characterization of ITO-Ag-ITO films on fibers by layer-by-layer method. Applied Surface Science, 2009, 255(19): 8197-8201.

[60] WANG X D, SUN D, DUAN Y Y, et al. Radiative characteristics of opacifier-loaded silica aerogel composites. Journal of Non-crystalline Solids, 2013, 375: 31-39.

[61] LEE S C, CUNNINGTON G R. Conduction and radiation heat transfer in high-porosity fiber thermal insulation. Journal of Thermophysics and Heat Transfer, 2000, 14(2): 121-136.

[62] TONG T W, SWATHI P S, CUNNINGTON JR G R. Examination of the radiative properties of coated silica fibers. Journal of Thermal Insulation, 1987, 11(1): 7-31.

[63] TONG T W, SWATHI P S, CUNNINGTON JR G R. Reduction of radiative heat transfer in thermal insulations by use of dielectric coated fibers. International Communications in Heat and Mass Transfer, 1989, 16(6): 851-860.

[64] HASS D D, PRASDA B D, GLASS D E, et al. Reflective Coating on Fibrous Insulation for Reduced Heat Transfer. NASA Contractor Report 201733, 1997.

[65] YANG L L, HE X, HE F. ITO coated quartz fibers for heat radiative applications. Materials Letters, 2008, 62(30): 4539-4541.

[66] GAN X, YU Z, YUAN K, et al. Preparation of a $\mathrm{CeO}_{2}$-nanoparticle thermal radiation shield coating on $\mathrm{ZrO}_{2}$ fibers via a hydrothermal method. Ceramics International, 2017, 43(16): 14183-14191.

[67] YANG J, ZHANG Y, HONG Z, et al. Preparations of $\mathrm{TiO}_{2}$ nanocrystal coating layers with various morphologies on mullite fibers for infrared opacifier application. Thin Solid Films, 2012, 520(7): 2651-2655.

[68] MA D, ZHU L, LIU B. Hydrothermally grown uniform $\mathrm{TiO}_{2}$ coatings on $\mathrm{ZrO}_{2}$ fibers and their infrared reflective and thermal conductive properties. Ceramics International, 2020, 46(3): 3400-3405.

[69] XU L, JIANG Y, FENG J, et al. Infrared-opacified $\mathrm{Al}_{2} \mathrm{O}_{3}-\mathrm{SiO}_{2}$ aerogel composites reinforced by $\mathrm{SiC}$-coated mullite fibers for thermal insulations. Ceramics International, 2015, 41(1): 437-442.

[70] WANG Y D, HAN C, ZHENG D, et al. Large-scale, flexible and high-temperature resistant $\mathrm{ZrO}_{2} / \mathrm{SiC}$ ultrafine fibers with a radial gradient composition. Journal of Materials Chemistry A, 2014, 2(25): 9607-9612. 